Rapid Reviews COVID-19

\title{
Review 1: "COVID-19 herd immunity in the Brazilian Amazon"
}

\section{Cesar Victora ${ }^{1}$}

${ }^{1}$ Universidade Federal de Pelotas, Programa de Pos-Graduacao em Epidemiologia, Brazil

Published on: Feb 07, 2021

License: Creative Commons Attribution 4.0 International License (CC-BY 4.0). 


\section{$\underline{\text { RR:C19 Evidence Scale rating by reviewer: }}$}

- Potentially informative. The main claims made are not strongly justified by the methods and data, but may yield some insight. The results and conclusions of the study may resemble those from the hypothetical ideal study, but there is substantial room for doubt. Decision-makers should consider this evidence only with a thorough understanding of its weaknesses, alongside other evidence and theory. Decision-makers should not consider this actionable, unless the weaknesses are clearly understood and there is other theory and evidence to further support it.

\section{Review:}

This paper is a welcome addition to the literature, as there is much to learn about how to assess population or herd immunity to SARS-CoV-2. The unknown parameters include the roles of cellular and humoral immunity, heterogeneity of transmission, the effects of social distancing and mask use on the subpopulation exposed to the virus, as well as what do diagnostic antibody tests really mean. Regarding the latter, further questions include how well antibody levels reflect immunity, how common are reinfections when there is a new viral challenge, how rapidly detectable antibodies disappear, and how often reinfections can occur.

The paper by Buss et al makes an important contribution. By studying antibody levels in blood donors over time in the city of Manaus, one of the most affected Brazilian cities, they corrected seroprevalence estimates by adjusting for antibody waning. This alone is an important contribution to the literature in light of mounting evidence of antibody declines. They conclude that about two thirds of the city population may have developed antibodies, and hypothesize that herd immunity has played a role in the drops in incidence and mortality observed in the city since the month of May.

Their study is rigorous and well presented. My concern as an epidemiologist is that extrapolating results obtained in blood donors to the whole city population may overestimate seroprevalence in the population. Antibody levels in household surveys in the Amazon region and throughout Brazil were higher in the age group from 20-59 years than in younger or older subjects.1 This limitation is duly acknowledged by the authors, who attempted to correct it by weighting results by age and sex to reproduce the structure of the city's population. Nevertheless, there are other characteristics of blood donors that may have biased the results, including the fact that they were apparently healthy, and possibly more likely to take risks than the rest of population. They were mobile, likely using public transportation in a high-prevalence city, not to speak of the fact that they may have been exposed 
during earlier visits to the blood collection center which is located near other hospitals. All of these factors may have contributed to higher exposure to SARS-CoV-2 than most of the city population.

The possibility that herd immunity has been reached is also challenged by the recent rise in cases in Manaus. Whereas in August the moving average was just over 200 reported cases a day, since midSeptember daily numbers have doubled. Death rates have also increased, although these remain low compared to the peak of the epidemic. Increased availability of testing is an alternative explanation for the rise in cases, but only time will tell whether the increase in deaths is sustained.

An alternative explanation is that high antibody prevalence, compatible with herd immunity, was reached in the past but reinfections are taking place - albeit at a much higher rate than has been suggested by the handful of cases reported so far in the literature.

Whether or not the hypothesis raised by Buss and colleagues - that herd immunity may have been reached in Manaus a few months ago - is proven to be correct, their article makes an important contribution to the literature, particularly regarding the interpretation and correction of seroprevalence estimates when detectable antibodies are waning over time. Their thorough analyses raise the question of whether or not herd immunity can be assessed with confidence, using existing tools.

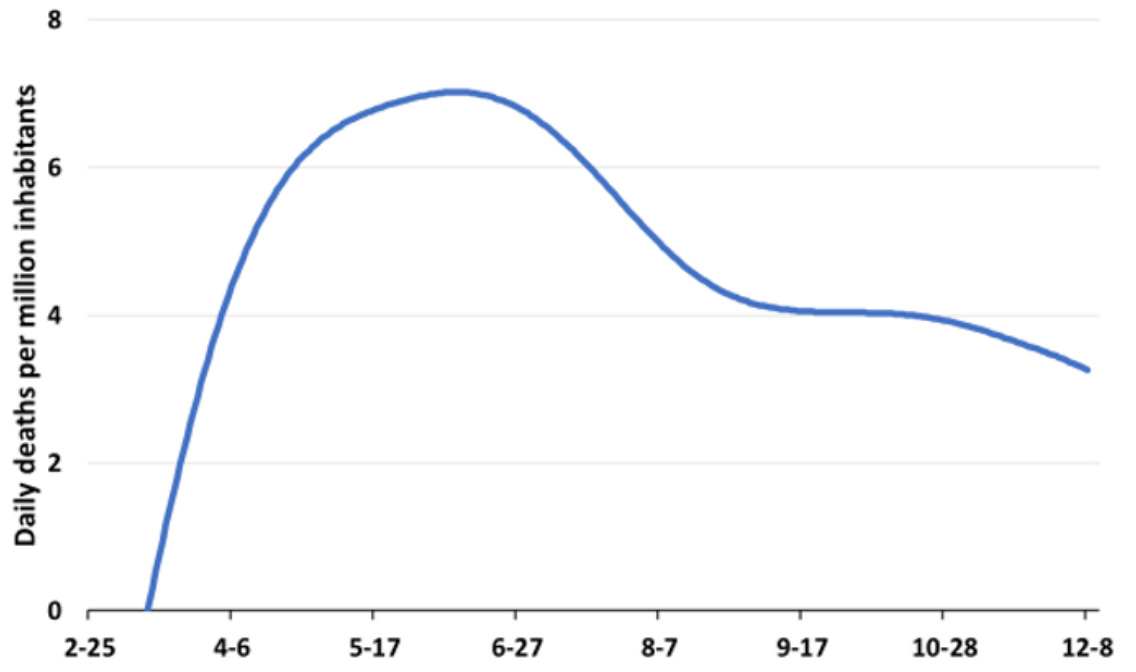

\section{Reference}

1. Hallal PC, Hartwig FP, Horta BL, et al. SARS-CoV-2 antibody prevalence in Brazil: results from two successive nationwide serological household surveys. Lancet Glob Health 2020. 